\title{
SHAPE CHARACTERIZATION OF MEDIASTINUM IN TUBERCULOSIS CHEST RADIOGRAPHS USING LEVEL SET SEGMENTATION
}

\author{
Sukanta Kumar Tulo', Satyavratan Govindarajan'1, Palaniappan Ramu², and \\ Ramakrishnan Swaminathan ${ }^{1}$ \\ ${ }^{1}$ Department of Applied Mechanics, ${ }^{2}$ Department of Engineering Design \\ Indian Institute of Technology Madras, Chennai, Tamil Nadu, India
}

\begin{abstract}
Corresponding Author: Sukanta Kumar Tulo
Non-Invasive Imaging and Diagnostics Laboratory, Biomedical Engineering Group, Department of Applied Mechanics, Indian Institute of Technology Madras, India 600036

Tel: +918249754096

Email: sukant.99t@gmail.com

https://doi.org/10.34107/BiomedSciInstrum.57.04212
\end{abstract}

\begin{abstract}
Mediastinum is considered as one of the substantial anatomical regions for the gross diagnosis of several chest related pathologies. The geometric variations of the mediastinum in Chest Radiographs (CXRs) could be utilised as potential image markers in the early detection of Tuberculosis (TB). This study attempts to segment mediastinum in CXRs using level sets for the shape characterization of TB conditions. The CXR images for this study are considered from a public database. An edgebased distance regularized level set evolution is employed to segment the lungs followed by a region-based Chan-Vese model that extracts mediastinum region. Features such as mediastinum area and lungs area are extracted from the segmented images. Further, mediastinum to lungs area ratio is calculated. Statistical analysis is performed on the features to differentiate normal and TB images. Results show that the proposed segmentation approach is able to segment the lungs and extract the mediastinum in CXRs. It is found that features namely mediastinum area and mediastinum to lungs area ratio are statistically significant in the differentiation of TB. Larger mediastinum area is observed in TB images as compared to normal. The performance of lung field segmentation is also observed to be in line with the literature. The mediastinum segmentation approach in CXRs obtains to be a novel method as compared to the existing methods. As the proposed approach based on mediastinum image analysis provides better shape characterization, the study could be clinically useful in the differentiation of TB conditions.
\end{abstract}

Keywords: Mediastinum, Tuberculosis, Chest radiographs, Level set, Shape characterization

\section{INTRODUCTION}

Tuberculosis (TB) is the world's leading infectious disease with 10 million cases reported in 2018, causing an estimated 1.5 million deaths [1]. Early detection of TB is essential for control and management of the disease. High-burden countries continue to have gaps in its diagnosis largely due to the lack of costeffective and rapid tools for screening. Diagnosis of TB using the gold standard, sputum culture testing is expensive and require several weeks for a conclusive result [2]. As the disease exhibits subtle and nonspecific abnormalities, there exists a challenge in its early detection [3].

Mediastinum is the thoracic compartment that is bounded by the lungs laterally, diaphragm inferiorly, thoracic inlet superiorly, sternum anteriorly, and posteriorly by the vertebral column. It houses many vital vascular and nonvascular organs and other structures including trachea, great vessels, heart and essential nerves [4]. The mediastinum is clinically significant due to the variety of structural abnormalities and functional pathologies that occur in this anatomic region. One of the possible sources of mediastinal abnormality is from the extension of the lungs or pleura [5]. TB manifestations such as airway enlargement, lymph node enlargement, pleura thickening and pleural effusions alters the structure of 